\title{
Study of Colored Pavement Application Design Based on Visual Perception Information
}

\author{
Shaoxin $\mathrm{Xi}^{1}$, Liangjie $\mathrm{Xu}^{1}$ \& Dong Chen ${ }^{1}$ \\ ${ }^{1}$ School of Transportation, Wuhan University of Technology, Hubei Wuhan, 430063, China
}

Keywords: colored pavement, visual, perception information, design.

\begin{abstract}
It becomes increasingly significant of rational design of colored pavement with its gradually applied in roads' construction. Under this circumstances, this dissertation firstly focuses on what psychological will impact on drivers by color influence, which is based on the driver's visual cognition combine with color psychology and perception psychology. Then proposes a special design method of colored pavement to rationally design of the stripes' separation distance, width and color. By this means, this paper intends to improve the influences of colored pavement the drivers' visual and then raise drivers' visibility and specificity when they get through the corner, meanwhile, to achieve the final goal of improving the accuracy of the driver's judgment and operation to ensure the most security of drivers.
\end{abstract}

\section{Introduction}

What drivers do is the vital elements to ensure the security when driving. During the driving, the driver mainly acquires information by judging the circumjacent visual information. Based on the circumjacent visual information, they can judge the vehicles' speed and position then make the judgments, decisions and actions. What the driver acquires is the visual information which accounted for over 90 percent [1]. It becomes increasingly functional of colored pavement in deceleration sections such as corners intersection with its gradually application in different roads [2]. Colored non-slip material can effectively deal with pavement of accident-prone area by increasing the friction between the tire and the pavement. Meanwhile, this material can also shorten braking distance, enhancing the vehicle's braking deceleration effect thus to avoid vicious traffic accidents.

\section{Colored Pavement Research Status}

There is rarely research of between structural features colored non-slip surface texture parameters and performance no matter at home or abroad $[3,6,7,8]$. In the field of the vision research colored pavement most researchers pay attention to the construction technology and materials against sliding properties. Therefore two cons of the researches of colored pavement visual design, and will be demonstrate as following:

(1)There are colored pavement application designs more casual, lack of support theory;

(2)At present, most of the researches are focusing on materials, pavement performance and construction technology. These kinds of researches pay their attention to varieties of the expression. Therefore there is rarely research in color pavement color combinations, layout spacing, color and other visual parameters and depending on the shape perception.

\section{Principles of Visual Perception Information}

Foreign scholars proposed two points from different aspects based on their researches: first, drivers pay most of the attention to the information range in $150 \mathrm{~m}$ when driving, especially to the distribution of pavement, which is from the aspect of distance of the gaze of view; second from the point of observation object, divers distribute $47.2 \%$ attention to the pavement when driving [4, 5]. It is an extremely complex process for the diver to accept outside visual stimulus then to produce a reaction and then do corresponding behavior. 
Driver gain information, processing that and then taking action accordingly, that is, driver's mental processes is a course of dealing with environmental information. When human eyes lightsensing cells converts the received light into nerve impulses, abstract the integration of the original signal received, then reorganization of the tens of thousands of received signals, convert them into boundary, towards more important visual information.

According to the researches of receptive field experiment [12], some complex cells belonging to visual cortex neurons can frequency maintain the response to lines and grating which is from a particular direction. As figure 1 shown, we clearly know that opposite movement stimulus is the strongest strong visual stimulation rather the other three directions that is homodromous, rightwards and leftwards movement. That is to say, highway pavement markings and stripes can cause strong visual stimulation. Therefore, it is meaningfully to rational design the colored pavement.

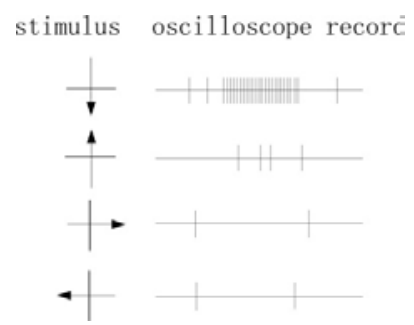

Fig.1: Sensitive to direction of complex cells receptive field

In the heart of scale, Stevens established the power law between physical quantities and feeling, shown as following formula:

$$
S=K I^{b}
$$

Here, $S$ is the quantity of feeling ; $K$ is a constant coefficient, which is related to specific feeling dimensions chosen units, such as inches, G; $I$ is the intensity of stimulation; $b$ is an index of the stimulus intensity, it is a constant for a special sensory dimensions.

For the light brightness, power exponent $b$ is about 0.33 .

The strength of sensory experience increases with increasing of light stimulation. That is to say, feeling is positively correlated with amount of stimulus intensity according to formula above. Therefore, it is a good way strengths drivers' alertness by appropriately increasing drivers' visual stimulation and enhancing feeling of visual changes.

\section{The Principle of Colored Pavement Color Recognition}

It is the most significant element of the driver in the view of the color is the most important visual information received in the driving environment, different wavelengths of light color to act on human's visual organ, it through the visual nerve to the brain, after thinking processing and forming a series of color psychological reaction. The driver's operation behavior easily swayed by emotions, color can cause effect of human mental and emotional lies in its reminiscent of things, including tense, active, dangerous, boring and so on.

In photoreceptors, cone cells are mainly responsible for the color discrimination and stimulation to distinguish subtle features of the day. Within the visible spectrum, the human eye to different wavelengths of light sensitivity, show in Figure 2, the curve on the right on behalf of the pyramidal cells of different wavelengths of sensibility, the left curve on behalf of the stem cells of the sensitivity of different cells. 


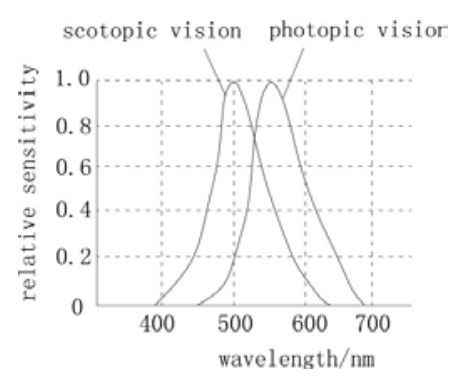

Fig.2: Light sensitive curve

Cone cells can absorb all the wavelengths of light, but the most sensitive to the intermediate spectra $(555 \mathrm{~nm})$, and for less than $500 \mathrm{~nm}$ or higher than $625 \mathrm{~nm}$ light sensitivity to poor. In the color selection of color pavement, with 500nm wavelength green light and the wavelength of 620nm red-orange light can cause more intense visual stimulation, by using the wavelength of $500 \mathrm{~nm}$ green light and wavelength of 620nm red-orange light, it can cause more strong visual stimulation and make drivers pay more attention on traffic environment.

\section{The Psychological Effect of Color Vision}

Visual color not only affects people's emotions, operation efficiency and life, but also affects the safe of drivers when driving. Colored pavement design mainly takes the advantages of color to create a good traffic environment condition, converts repetitive mental and physical stimulation to a strongly physiological reaction, which may give a reminding to both drivers and other traffic participants to pay more attention to observe the traffic environment. Different colors brings out different sense of touch, such as weight, feeling temperature, and causes people to produce the psychological effects such as excitement, tension, irritability.

It is very monotonous for the color of pavement at present, for most of the color applied is gray or black. From the perspective of the psychology of color, black and gray often makes people feel upset, disappointed, darkly and lonely which is a negative state. Therefore, it will produce visual psychological depression for driver. Rationally takes the advantages of the psychological effects of color on people, designed a unique color thus to improve the gray pavement, which can make people travel more comfortable and safer.

On the selection of pavement color, white can make people feel calm, green can alleviate the human nervous, blue can make people calm and can inhibit a person too excited mood, red can make people's psychological activity, yellow make people have be jubilant mood. But on the curve, pay more attention to the color caused by the attention and danger signal transmission, red and yellow light penetrating is stronger, and it have better warning effect to give people a strong visual stimulation, so the driver timely reflect and make the operation of driving behavior accordingly.

\section{Design of Color Pavement}

Stripe Spacing Design of Color Pavement.The main idea of designing width between two stripes is to gradually decrease the distance between two adjacent fringes along the direction of travel. Using the illusion of time produced by the fringe spacing is approximately equal to gradually reduce the running speed.

Calculation process of colored pavement deceleration stripe spacing shown as following:

$$
a=\frac{d v}{d t}
$$

Where, $v$ is the velocity of the vehicle, $(\mathrm{m} / \mathrm{s}) ; a$ is the vehicle deceleration, $\left(\mathrm{m} / \mathrm{s}^{2}\right) ; t$ is the time, (s).

It can further derivation for formula 3:

$$
\int_{V_{t}}^{V_{t+\Delta t}} d v=\int_{t}^{t+\Delta t} a d v
$$


And further calculation formula is:

$$
v_{t+\Delta t}-v_{t}=a \Delta t
$$

Here, $\Delta t$ representation of the time step, it is related with the stripe number and blink rate.

And then the calculation formula of $\Delta t$ is:

$$
\Delta t=\frac{n}{f}
$$

So, the calculation formula of $v_{t+\Delta t}$ is:

$$
v_{t+\Delta t}=v_{t}+a \frac{n}{f}
$$

Each color pavement deceleration stripe position can be calculated by the following formula:

$$
\frac{d x}{d t}=v_{0}+a \Delta t
$$

Where, $x$ is the distance from the starting position to the position of color stripe, (m), shown as figure $3 ; v_{0}$ is the speed of the vehicle when entering the deceleration region, $(\mathrm{m} / \mathrm{s})$.

It can further derivation formula, that is:

$$
\int_{0}^{t} d x=\int_{0}^{t}\left(\mathrm{at}+\mathrm{v}_{0}\right) d t
$$

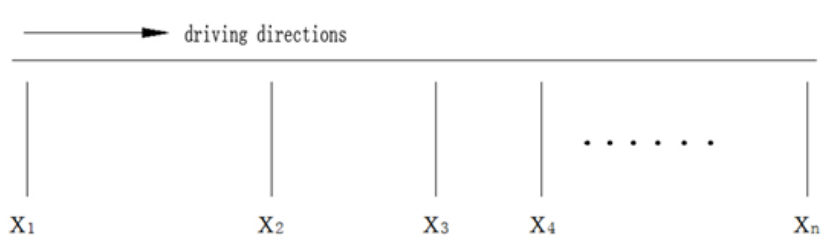

Fig.3: Color pavement deceleration fringe location diagram

So it can get colored pavement slow stripe position calculation formula is:

$$
x_{t}=\frac{1}{2} a t^{2}+v_{0} t+x_{0}
$$

Combining equation (5), resulting in colored pavement stripes deceleration position calculation models:

$$
x_{t}=\frac{1}{2} a\left(\frac{n}{f}\right)^{2}+v_{0}\left(\frac{n}{f}\right)+x_{0}
$$

Where, $x_{0}$ is the position of the first stripes, usually take zero, (m).

Combined with the actual situation of road, the selected vehicles' initial velocity when enter deceleration area and blink rate, we can calculate the of distance $n$ line related to $x_{0}$. Colored pavement fringe spacing can be calculated through the fringe position, the formula is:

$$
s_{n}=x_{t+1}-x_{t}
$$

The design of deceleration stripe spacing should take the driver's comfort into consideration in the terms of the selection of the width stripe. Traffic Engineering Manual shows that when the speed of deceleration achieving $3 \mathrm{~m} / \mathrm{s}^{2}$ people still feel comfortable. That is, when speed of deceleration is between $0 \mathrm{~m} / \mathrm{s}^{2}$ to $3 \mathrm{~m} / \mathrm{s}^{2}$ it is acceptable.

In order to simplifying the calculation process, facilitating easily road construction, preventing driver emergency braking this paper chooses $2.5 \mathrm{~m} / \mathrm{s}^{2}$ as deceleration speed in the calculation of the width of stripes. According to Allen [12], form Australia flash it is accepted if the flash frequency no more than 4 times per second.

We assume the speed of vehicle is $60 \mathrm{~km} / \mathrm{h}$ when driving into the color pavement deceleration section. The selected speed of deceleration is $-2.5 \mathrm{~m} / \mathrm{s}^{2}$ in order to prevent emergency braking. Flash frequency is $4 \mathrm{bps}$. Therefore, this paper determines the design value of the stripe spacing of color pavement is shown in table 1 . 
Table 1: Color pavement transition section of the fringe spacing design value

\begin{tabular}{lllllllllll}
\hline $\begin{array}{l}\text { Spacing } \\
\text { No. }\end{array}$ & S1 & S2 & S3 & S4 & S5 & S6 & S7 & S8 & S9 & S10 \\
\hline $\begin{array}{l}\text { Spacing } \\
\text { length }\end{array}$ & 3.9 & 3.8 & 3.6 & 3.5 & 3.3 & 3.2 & 3.0 & 2.8 & 2.7 & 2.5 \\
\hline
\end{tabular}

Stripe Width Design of Color Pavement.The main idea of colored pavement stripe width design is that tries every effect to have the contemplation of driver's consistent stay in the color stripes in every stripe. The calculation of the time that drivers stay in colored pavement is shown as following formula:

$$
d t=\frac{d s}{d v}
$$

It can be seen form the above formula, corresponding stripe width is proportional to the speed when contemplation time is constant. Driver given a false sense of accelerated by applying rules of gradually decreasing of the width. Thus it can remind the driver to slowing down before entering the curve. On the selection of stripe width, considering the reality of colored pavement selection of stripe width of 2meters, this research set up the first striped width of 3meters and 0.2meters under a reduced, forming a regular decreasing gradient.

\section{Practical Application of Colored Pavement Design}

The curve of color pavement design. In this paper, using color pavement color effect and sliding characteristics of reasonable design of color pavement, it can effectively reduce the occurrence of traffic accidents. In the design of color pavement, with two-way four lane highway curve, for example, in order to improve the road surface adhesion of vehicle during cornering, in the corners before and after 25meters all covered with colored pavement, and outside the 25meters using deceleration gradual transition zone, pavement color to red and orange color is better, the specific design as shown in figure 4.

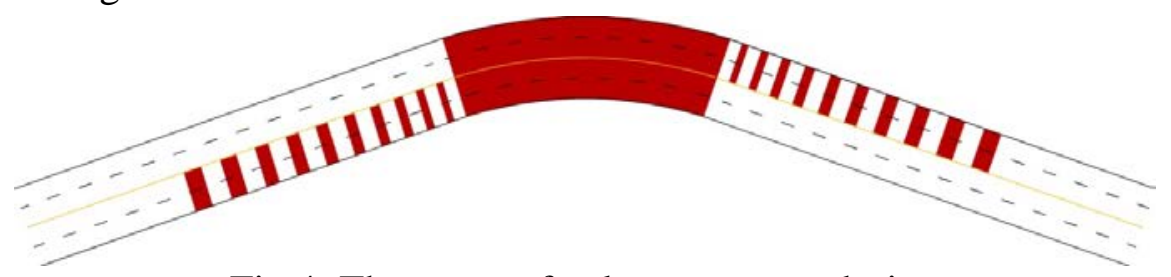

Fig.4: The curve of color pavement design

Color pavement design of intersection without signal control.In many on signal control intersection, most traffic accidents are due to excessive speed. T-intersection, for example, laying colored pavement in front of intersection stop line can alert the driver slow down.

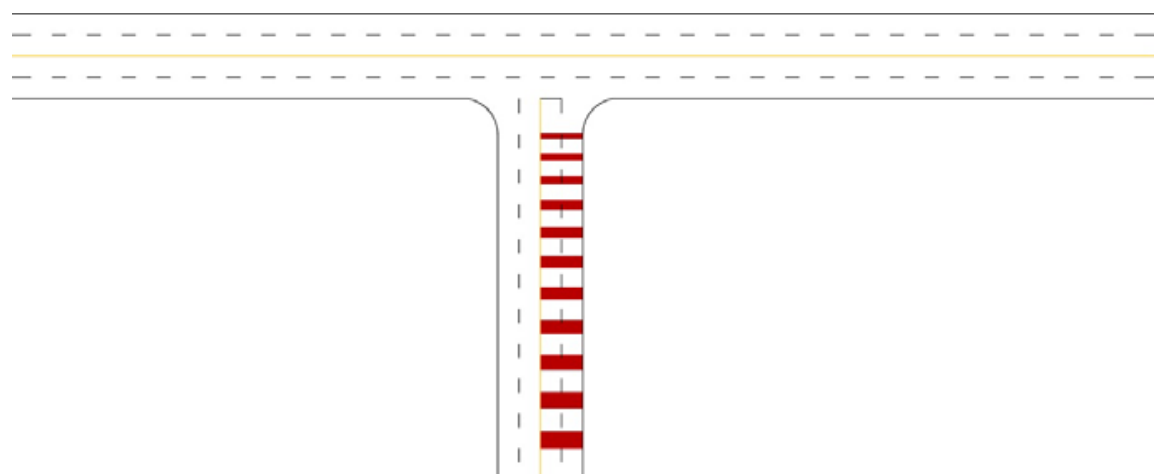

Fig.5: Color pavement design of T- intersection

The purpose of the application of colored pavement design is to alert the driver's attention in front of the dangerous road, to remind the driver to observe traffic environment and control the vehicle to slow down in advance, the theoretical deceleration of this design is $2.5 \mathrm{~m} / \mathrm{s}^{2}$. 


\section{Conclusions}

It becomes increasingly significant of rational design of colored pavement with its gradually applied in roads' construction. This dissertation firstly focuses on what psychological will the drivers have by impacting on color, which is based on the driver's visual cognition combined with color psychology and perception psychology. Then proposes a special design means of colored pavement to achieve the goal that rationally design of the stripes' separation distance, width and color of colored pavement. Meanwhile, taking curve and T-intersection as example to rationally design color pavement thus to sound apply to road construction.

Red and orange-yellow is the best choice to colored pavement, the design of stripe spacing and width of stripe be gradually reduced thus to ensure the color do best effect of reminding drivers.

It takes the advantage of drivers' visual illusion to gradually reduce the speed of vehicle. At the same time, through the color pavement design gives the driver strong visual impact stimulation, this will increase the driver running on curve of specificity and legibility. This design allows the driver to produce a strong psychological alertness, improve the operation of the driver's judgment and accuracy, and ultimately to improve road safety effects.

\section{References}

[1] Zhou Xiaobo. Approach and application of steering on freeway curve based on visual information. Wuhan University of Technology, 2010

[2] Zhao An'ping, Tang Ai'ping, Wang Wei. Material Properties and Application of Colored Antiskid Pavement. Road Machinery \& Construction Mechanization.2013, 30(2)

[3] Yan Xianhua. Study on Design of Highway Optical Illusion Deceleration Markings, Chang'an University, 2011

[4] P.Simon, J-C.Popieul, P.Loslever, P.Leroux and D.Sonnerat. Car Drivers Eye Scanning Behaviour During A Monotonous Journey on Highway, Car Drivers Eye Scanning Behaviour, 2005

[5] K.Takemura J.Ido Y.Matsumoto T.Ogasawar, Drive Monitoring System Based on NonContact Measurement System of Driver's Focus of Visual Attention

[6] Xu, Ming, Pan, Xiaodong, Deng, Qi ,Setting method of thin-layer antiskid colored pavement in tunnel based on increasing luminance of pavement, Engineering Village, (2012)3073-3083

[7] WANG Jianwen. New Colored Antiskid Material Technology Application in Highway Project. Communications Standardization, 2011,Z1:184-186.

[8] José M. Pardillo Mayora, Rafael Jurado Pi na. An assessment of the skid resistance effect on traffic safety under wet-pavement conditions. Elsevier Accident Analysis and Prevention 41 (2009) 881-886.

[9] XU Hongliang, ZHANG Taixiong, TANG Boming, LU Tangzhi. Design of Road Traffic Safety Color System Based on Color Perception, Journal of chongqing jiaotong university (natural science edition). 2009, 28(1)

[10] GAO Xiangping. psychology of perception. People's Education Press, 2011, PP56-90.

[11] LIU Haoxue. traffic psychology. Shaanxi science and Technology Press, 1992 